\title{
[1] AN INDEX TO FREY'S JEWISH INSCRIPTIONS IN RECENT NEW EDITIONS
}

\author{
David Lincicum*
}

\begin{abstract}
This index indicates which inscriptions in J.-B. Frey's Corpus Inscriptionum Iudaicarum (CIf), long seen to be in need of revision, have been re-edited in the six excellent volumes of Jewish inscriptions published recently in two series by Cambridge University Press and Mohr Siebeck in Tübingen. Though each of these volumes has its own index to Frey's corpus, to combine them here may facilitate ease of reference, especially helpful in evaluating older works which make reference to inscriptions by Frey's numbers.
\end{abstract}

The modest purpose of this index is to indicate which inscriptions in J.-B. Frey's Corpus Inscriptionum Iudaicarum $(C I f),{ }^{1}$ long seen to be in need of revision, have been re-edited in the six excellent volumes of Jewish inscriptions published recently in two series by Cambridge University Press and Mohr Siebeck in Tübingen. Though each of these volumes has its own index to Frey's corpus, to combine them here may facilitate ease of reference, especially helpful in evaluating older works which make reference to inscriptions by Frey's numbers. Of course, this is only a provisional index, and it is to be hoped that once the Corpus Inscriptionum fudaeae/Palaestinae project has been completed, a comprehensive index to Jewish inscriptions including much more information will be made available. ${ }^{2}$

The following abbreviations are here used:

FIGRE William Horbury and David Noy, Jewish Inscriptions of Graeco-Roman Egypt (Cambridge and New York: Cambridge University Press, 1992).

FIWE 1-2 David Noy, Fewish Inscriptions of Western Europe. Volume I: Italy (Excluding the City of Rome), Spain and Gaul; Volume II: The City of Rome (Cambridge: Cambridge University Press, 1993, 1995).

IfudO 1-3 Inscriptiones Fudaicae Orientis: David Noy, Alexander Panayotov, and Hanswulf Bloedhorn, Volume 1: Eastern Europe (TSAJ 101; Tübingen: Mohr Siebeck, 2004); Walter Ameling, Band 2: Kleinasien (TSAJ 99; Tübingen: Mohr Siebeck, 2004); David Noy and Hanswulf Bloedhorn, Volume 3: Syria and Cyprus (TSAJ 102; Tübingen: Mohr Siebeck, 2004).

\footnotetext{
* D.Phil. Candidate, Keble College, University of Oxford. Email: david.lincicum@theology.ox.ac.uk.

${ }^{1}$ J.-B. Frey, Corpus Inscriptionum Iudaicarum. Recueil des inscriptions juives qui vont du IIIe siècle avant fésus-Christ au VIIe siècle de notre ère (2 vols.; Rome: Pontificio Istituto di Archeologia Cristiana, 1936, 1952). Volume 1 was reissued with a substantial "Prolegomenon" by B. Lifshitz (New York: Ktav, 1975), and his additions are included in this index.

${ }^{2}$ For the project announcement, see Hannah M. Cotton, Leah Di Segni, Werner Eck and Benjamin Isaac, "Corpus Inscriptionum Judaeae/Palaestinae", ZPE 127 (1999): 307-08.
} 


\begin{tabular}{|c|c|c|c|}
\hline CIf \# & New Edition & 44 & JIWE 2.431 \\
\hline 1 & JIWE 2.556 & 45 & JIWE 2.525 \\
\hline 2 & JIWE 2.530 & 46 & JIWE 2.468 \\
\hline 3 & fIWE 2.531 & 47 & JIWE 2.486 \\
\hline 4 & JIWE 2.532 & 48 & JIWE 2.443 \\
\hline 5 & JIWE 2.626.i & 49 & JIWE 2.492 \\
\hline 6 & JIWE 2.437 & 50 & JIWE 2.429 \\
\hline 7 & JIWE 2.436 & 51 & JIWE 2.460 \\
\hline 8 & JIWE 2.461 & 52 & JIWE 2.485 \\
\hline 9 & JIWE 2.487 & 53 & JIWE 2.484 \\
\hline 10 & JIWE 2.466 & 54 & fIWE 2.493 \\
\hline 11 & JIWE 2.440 & 55 & JIWE 2.463 \\
\hline 12 & JIWE 2.481 & 56 & JIWE 2.475 \\
\hline 13 & JIWE 2.480 & 57 & JIWE 2.425 \\
\hline 14 & JIWE 2.523 & 58 & JIWE 2.448 \\
\hline 15 & JIWE 2.442 & 59 & JIWE 2.470 \\
\hline 16 & JIWE 2.441 & 60 & JIWE 2.434 \\
\hline 17 & fIWE 2.471 & 61 & JIWE 2.478 \\
\hline 18 & JIWE 2.428 & 62 & JIWE 2.477 \\
\hline 19 & JIWE 2.456 & 63 & JIWE 2.500 \\
\hline 20 & JIWE 2.435 & 64 & JIWE 2.430 \\
\hline 21 & JIWE 2.489 & 65 & JIWE 2.464 \\
\hline 22 & JIWE 2.451 & 66 & JIWE 2.413 \\
\hline 23 & JIWE 2.450 & 67 & JIWE 2.452 \\
\hline 24 & JIWE 2.473 & 68 & JIWE 2.491 \\
\hline 25 & JIWE 2.459 & 69 & JIWE 2.416 \\
\hline 26 & JIWE 2.467 & 70 & JIWE 2.494 \\
\hline 27 & JIWE 2.453 & 71 & JIWE 2.495 \\
\hline 28 & JIWE 2.483 & 72 & JIWE 2.616 \\
\hline 29 & JIWE 2.469 & 73 & JIWE 2.p.337 \\
\hline 30 & JIWE 2.454 & 74 & JIWE 2.p.337 \\
\hline 31 & JIWE 2.457 & 75 & JIWE 2.407 \\
\hline 32 & JIWE 2.474 & 76 & JIWE 2.409 \\
\hline 33 & JIWE 2.444 & 77 & JIWE 2.408 \\
\hline 34 & JIWE 2.524 & 78 & JIWE 2.533 \\
\hline 35 & JIWE 2.479 & 79 & JIWE 2.628.i \\
\hline $35 \mathrm{a}$ & JIWE 2.527 & 80 & JIWE 2.628.ii \\
\hline 36 & JIWE 2.526 & 81 & JIWE 2.324 \\
\hline 37 & JIWE 2.488 & 82 & JIWE 2.350 \\
\hline 38 & JIWE 2.482 & 83 & JIWE 2.206 \\
\hline 39 & JIWE 2.455 & 84 & JIWE 2.340 \\
\hline 40 & JIWE 2.449 & 85 & JIWE 2.259 \\
\hline 41 & JIWE 2.458 & 86 & JIWE 2.276 \\
\hline 42 & JIWE 2.462 & 87 & JIWE 2.242 \\
\hline 43 & JIWE 2.490 & 88 & JIWE 2.288 \\
\hline
\end{tabular}




\begin{tabular}{|c|c|c|c|}
\hline 89 & JIWE 2.382 & 134 & JIWE 2.228 \\
\hline 90 & JIWE 2.334 & 135 & fIWE 2.327 \\
\hline 91 & JIWE 2.317 & 136 & FIWE 2.205 \\
\hline 92 & fIWE 2.336 & 137 & JIWE 2.222 \\
\hline 93 & JIWE 2.209 & 138 & JIWE 2.232 \\
\hline 94 & JIWE 2.304 & 139 & FIWE 2.208 \\
\hline 95 & JIWE 2.351 & 140 & FIWE 2.338 \\
\hline 96 & JIWE 2.353 & 141 & JIWE 2.345 \\
\hline 97 & JIWE 2.333 & 142 & JIWE 2.263 \\
\hline 98 & JIWE 2.219 & 143 & FIWE 2.346 \\
\hline 99 & JIWE 2.255 & 144 & JIWE 2.246 \\
\hline 100 & JIWE 2.373 & 145 & JIWE 2.257 \\
\hline 101 & JIWE 2.306 & 146 & FIWE 2.256 \\
\hline 102 & JIWE 2.250 & 147 & fIWE 2.238 \\
\hline 103 & JIWE 2.236 & 148 & fIWE 2.253 \\
\hline 104 & JIWE 2.393 & 149 & FIWE 2.223 \\
\hline 105 & JIWE 2.205a & 150 & JIWE 2.329 \\
\hline 106 & fIWE 2.321 & 151 & FIWE 2.347 \\
\hline 107 & JIWE 2.294 & 152 & JIWE 2.254 \\
\hline 108 & JIWE 2.551 & 153 & JIWE 2.339 \\
\hline 109 & JIWE 2.277 & 154 & FIWE 2.227 \\
\hline 110 & JIWE 2.235 & 155 & fIWE 2.244 \\
\hline 111 & JIWE 2.212 & 156 & JIWE 2.356 \\
\hline 112 & JIWE 2.229 & 157 & JIWE 2.269 \\
\hline 113 & JIWE 2.374 & 158 & JIWE 2.376 \\
\hline 114 & JIWE 2.309 & 159 & JIWE 2.348 \\
\hline 115 & JIWE 2.299 & 160 & JIWE 2.295 \\
\hline 116 & JIWE 2.315 & 161 & JIWE 2.274 \\
\hline 117 & JIWE 2.213 & 162 & JIWE 2.268 \\
\hline 118 & JIWE 2.342 & 163 & FIWE 2.305 \\
\hline 119 & JIWE 2.354 & 164 & fIWE 2.383 \\
\hline 120 & JIWE 2.337 & 165 & JIWE 2.310 \\
\hline 121 & JIWE 2.231 & 166 & FIWE 2.251 \\
\hline 122 & JIWE 2.262 & 167 & fIWE 2.239 \\
\hline 123 & JIWE 2.326 & 168 & JIWE 2.357 \\
\hline 124 & JIWE 2.267 & 169 & JIWE 2.358 \\
\hline 125 & JIWE 2.344 & 170 & fIWE 2.394 \\
\hline 126 & JIWE 2.282 & 171 & fIWE 2.272 \\
\hline 127 & JIWE 2.293 & 172 & JIWE 2.290 \\
\hline 128 & JIWE 2.316 & 173 & JIWE 2.292 \\
\hline 129 & JIWE 2.237 & 174 & FIWE 2.397 \\
\hline 130 & JIWE 2.355 & 175 & FIWE 2.300 \\
\hline 131 & JIWE 2.375 & 176 & fIWE 2.211 \\
\hline 132 & JIWE 2.281 & 177 & JIWE 2.243 \\
\hline 133 & JIWE 2.286 & 178 & FIWE 2.384 \\
\hline
\end{tabular}




\begin{tabular}{|c|c|c|c|}
\hline 179 & JIWE 2.230 & 224 & JIWE 2.379 \\
\hline 180 & JIWE 2.255 & 225 & JIWE 2.249 \\
\hline 181 & JIWE 2.303 & 226 & JIWE 2.247 \\
\hline 182 & JIWE 2.385 & 227 & JIWE 2.312 \\
\hline 183 & JIWE 2.386; IfudO 3.p.116 & 228 & JIWE 2.207 \\
\hline 184 & JIWE 2.280 & 229 & JIWE 2.204 \\
\hline 185 & fIWE 2.387 & 230 & JIWE 2.291 \\
\hline 186 & fIWE 2.257 & 231 & JIWE 2.283 \\
\hline 187 & JIWE 2.398 & 232 & JIWE 2.359 \\
\hline 188 & JIWE 2.388 & 233 & JIWE 2.361 \\
\hline 189 & fIWE 2.389 & 234 & JIWE 2.323 \\
\hline 190 & JIWE 2.390 & 235 & JIWE 2.273 \\
\hline 191 & JIWE 2.221 & 236 & JIWE 2.367 \\
\hline 192 & JIWE 2.216 & 237 & JIWE 2.258 \\
\hline 193 & fIWE 2.270 & 238 & JIWE 2.296 \\
\hline 194 & JIWE 2.320 & 239 & JIWE 2.368 \\
\hline 195 & JIWE 2.362 & 240 & JIWE 2.369 \\
\hline 196 & JIWE 2.240 & 241 & JIWE 2.370 \\
\hline 197 & JIWE 2.314 & 242 & JIWE 2.308 \\
\hline 198 & JIWE 2.215 & 243 & fIWE 2.371 \\
\hline 199 & JIWE 2.395 & 244 & JIWE 2.319 \\
\hline 200 & JIWE 2.331 & 245 & JIWE 2.260 \\
\hline 201 & JIWE 2.307 & 246 & JIWE 2.330 \\
\hline 202 & JIWE 2.392 & 247 & JIWE 2.380 \\
\hline 203 & JIWE 2.240 & 248 & JIWE 2.335 \\
\hline 204 & JIWE 2.301 & 249 & fIWE 2.261 \\
\hline 205 & JIWE 2.302 & 250 & JIWE 2.233 \\
\hline 206 & JIWE 2.217 & 251 & JIWE 2.629.i \\
\hline 207 & JIWE 2.264 & 252 & JIWE 2.271 \\
\hline 208 & JIWE 2.285 & 253 & JIWE 2.297 \\
\hline 209 & JIWE 2.325 & 254 & JIWE 2.248 \\
\hline 210 & JIWE 2.343 & 255 & JIWE 2.298 \\
\hline 211 & JIWE 2.245 & 256 & JIWE 2.218 \\
\hline 212 & JIWE 2.377 & 257 & JIWE 2.275 \\
\hline 213 & JIWE 2.278 & 258 & JIWE 2.313 \\
\hline 214 & fIWE 2.241 & 259 & JIWE 2.366 \\
\hline 215 & JIWE 2.328 & 260 & JIWE 2.234 \\
\hline 216 & JIWE 2.265 & 261 & JIWE 2.311 \\
\hline 217 & JIWE 2.284 & 262 & JIWE 2.352 \\
\hline 218 & fIWE 2.226 & 263 & JIWE 2.220 \\
\hline 219 & JIWE 2.279 & 264 & JIWE 2.332 \\
\hline 220 & JIWE 2.378 & 265 & JIWE 2.322 \\
\hline 221 & fIWE 2.266 & 266 & JIWE 2.287 \\
\hline 222 & fIWE 2.224 & 267 & JIWE 2.381 \\
\hline 223 & JIWE 2.318 & 268 & JIWE 2.349 \\
\hline
\end{tabular}




\begin{tabular}{|c|c|c|c|}
\hline 269 & JIWE 2.289 & 314 & JIWE 2.172 \\
\hline 270 & JIWE 2.391 & 315 & FIWE 2.11 \\
\hline 271 & JIWE 2.210 & 316 & FIWE 2.98 \\
\hline 272 & JIWE 2.399 & 317 & FIWE 2.2 \\
\hline 273 & JIWE 2.214 & 318 & FIWE 2.114 \\
\hline 274 & JIWE 2.225 & 319 & FIWE 2.560 \\
\hline 275 & JIWE 2.396 & 320 & FIWE 2.59 \\
\hline 276 & JIWE 2.308 & 321 & FIWE 2.171 \\
\hline 277 & JIWE 2.402 & 322 & FIWE 2.32 \\
\hline 278 & JIWE 2.403 & 323 & FIWE 2.16 \\
\hline 279 & JIWE 2.404 & 324 & JIWE 2.121 \\
\hline 280 & JIWE 2.405 & 325 & FIWE 2.101 \\
\hline 281 & JIWE 2.406 & 326 & FIWE 2.116 \\
\hline 282 & JIWE 2.534 & 327 & FIWE 2.185 \\
\hline 283 & JIWE 2.535 & 328 & FIWE 2.111 \\
\hline 284 & JIWE 2.547 & 329 & FIWE 2.22 \\
\hline 285 & JIWE 2.626.ii & 330 & fIWE 2.17 \\
\hline 286 & JIWE 2.536 & 331 & JIWE 2.6 \\
\hline 287 & JIWE 2.612 & 332 & FIWE 2.168 \\
\hline 288 & JIWE 2.537 & 333 & FIWE 2.68 \\
\hline 289 & JIWE 2.538 & 334 & FIWE 2.93 \\
\hline 290 & JIWE 2.58 & 335 & FIWE 2.99 \\
\hline 291 & FIWE 2.33 & 336 & FIWE 2.13 \\
\hline 292 & fIWE 2.53 & 337 & FIWE 2.164 \\
\hline 293 & JIWE 2.92 & 338 & FIWE 2.169 \\
\hline 294 & fIWE 2.47 & 339 & fIWE 2.83 \\
\hline 295 & FIWE 2.203.xviii \& 153 & 340 & JIWE 2.50 \\
\hline 296 & FIWE 2.183; IfudO 2.212 & 341 & FIWE 2.34 \\
\hline 297 & FIWE 2.14 & 342 & FIWE 2.115 \\
\hline 298 & JIWE 2.173 & 343 & FIWE 2.167 \\
\hline 299 & JIWE 2.119 & 344 & FIWE 2.123 \\
\hline 300 & fIWE 2.15 & 345 & FIWE 2.152 \\
\hline 301 & JIWE 2.96 & 346 & FIWE 2.80 \\
\hline 302 & FIWE 2.79 & 347 & FIWE 2.124 \\
\hline 303 & JIWE 2.46 & 348 & FIWE 2.41 \\
\hline 304 & JIWE 2.69 & 349 & JIWE 2.186 \\
\hline 305 & FIWE 2.8 & 350 & FIWE 2.152 \\
\hline 306 & JIWE 2.91 & 351 & FIWE 2.188 \\
\hline 307 & JIWE 2.184 & 352 & FIWE 2.27 \\
\hline 308 & JIWE 2.12 & 353 & FIWE 2.86 \\
\hline 309 & JIWE 2.20 & 354 & FIWE 2.44 \\
\hline 310 & JIWE 2.120 & 355 & FIWE 2.125 \\
\hline 311 & JIWE 2.82 & 356 & FIWE 2.70 \\
\hline 312 & JIWE 2.563 & 357 & FIWE 2.71 \\
\hline 313 & JIWE 2.54 & 358 & JIWE 2.25 \\
\hline
\end{tabular}




\begin{tabular}{|c|c|c|c|}
\hline 359 & JIWE 2.126 & 404 & JIWE 2.159 \\
\hline 360 & JIWE 2.23 & 405 & JIWE 2.162 \\
\hline 361 & FIWE 2.102 & 406 & fIWE 2.45 \\
\hline 362 & JIWE 2.60 & 407 & JIWE 2.36 \\
\hline 363 & FIWE 2.127 & 408 & fIWE 2.113 \\
\hline 364 & FIWE 2.39 & 409 & fIWE 2.55 \\
\hline 365 & FIWE 2.170 & 410 & fIWE 2.66 \\
\hline 366 & FIWE 2.75 & 411 & FIWE 2.10 \\
\hline 367 & FIWE 2.567 & 412 & JIWE 2.107 \\
\hline 368 & FIWE 2.189 & 413 & JIWE 2.175 \\
\hline 369 & FIWE 2.28 & 414 & fIWE 2.26 \\
\hline 370 & JIWE 2.112 & 415 & fIWE 2.51 \\
\hline 371 & JIWE 2.94 & 416 & JIWE 2.194 \\
\hline 372 & FIWE 2.190 & 417 & JIWE 2.163 \\
\hline 373 & FIWE 2.157 & 418 & fIWE 2.118 \\
\hline 374 & FIWE 2.56 & 419 & fIWE 2.63 \\
\hline 375 & FIWE 2.109 & 420 & JIWE 2.200 \\
\hline 376 & FIWE 2.18 & 421 & JIWE 2.129 \\
\hline 377 & FIWE 2.128 & 422 & fIWE 2.49 \\
\hline 378 & JIWE 2.176 & 423 & JIWE 2.177 \\
\hline 379 & JIWE 2.108 & 424 & JIWE 2.29 \\
\hline 380 & FIWE 2.557 & 425 & JIWE 2.130 \\
\hline 381 & FIWE 2.191 & 426 & fIWE 2.21 \\
\hline 382 & FIWE 2.192 & 427 & FIWE 2.77 \\
\hline 383 & FIWE 2.117 & 428 & FIWE 2.203.v \\
\hline 384 & JIWE 2.165 & 429 & fIWE 2.131 \\
\hline 385 & FIWE 2.548 & 430 & JIWE 2.57 \\
\hline 386 & FIWE 2.30 & 431 & JIWE 2.132 \\
\hline 387 & fIWE 2.35 & 432 & fIWE 2.90 \\
\hline 388 & FIWE 2.38 & 433 & fIWE 2.1 \\
\hline 389 & JIWE 2.72 & 434 & fIWE 2.81 \\
\hline 390 & JIWE 2.166 & 435 & JIWE 2.89 \\
\hline 391 & FIWE 2.110 & 436 & JIWE 2.133 \\
\hline 392 & FIWE 2.9 & 437 & JIWE 2.42 \\
\hline 393 & JIWE 2.76 & 438 & fIWE 2.134 \\
\hline 394 & FIWE 2.19 & 439 & JIWE 2.135 \\
\hline 395 & FIWE 2.47 & 440 & JIWE 2.178 \\
\hline 396 & FIWE 2.3 & 441 & JIWE 2.95 \\
\hline 397 & FIWE 2.193 & 442 & JIWE 2.168 \\
\hline 398 & FIWE 2.106 & 443 & fIWE 2.136 \\
\hline 399 & FIWE 2.174 & 444 & 7IWE 2.65 \\
\hline 400 & FIWE 2.24 & 445 & JIWE 2.158 \\
\hline 401 & JIWE 2.187 & 446 & fIWE 2.154 \\
\hline 402 & JIWE 2.100 & 447 & fIWE 2.137 \\
\hline 403 & JIWE 2.52 & 448 & JIWE 2. 203.xxxix \\
\hline
\end{tabular}




\begin{tabular}{|c|c|c|c|}
\hline 449 & JIWE 2.138 & $493 \mathrm{a}$ & FIWE 2.203.xlii \\
\hline 450 & JIWE 2.31 & 494 & FIWE 2.540 \\
\hline 451 & FIWE 2.139 & 495 & JIWE 2.541 \\
\hline 452 & fIWE 2.203.xl & 496 & JIWE 2.542 \\
\hline 453 & JIWE 2.64 & 497 & JIWE 2.539 \\
\hline 454 & fIWE 2.73 & 498 & fIWE 2.573 \\
\hline 455 & JIWE 2.37 & 499 & fIWE 2.550 \\
\hline 456 & JIWE 2.85 & 500 & fIWE 2.627.i \\
\hline 457 & JIWE 2.179 & 501 & JIWE 2.568; IfudO 3.Syr33 \\
\hline 458 & JIWE 2.196 & 502 & JIWE 2.561 \\
\hline 459 & JIWE 2.97 & 503 & JIWE 2.549 \\
\hline 460 & JIWE 2.195 & 504 & FIWE 2.558 \\
\hline 461 & JIWE 2.181 & 505 & FIWE 2.559 \\
\hline 462 & JIWE 2.62 & 506 & JIWE 2.569 \\
\hline 463 & FIWE 2.43 & 507 & FIWE 2.543 \\
\hline 464 & JIWE 2.608 & 508 & JIWE 2.544 \\
\hline 465 & JIWE 2.4 & 509 & JIWE 2.576 \\
\hline 466 & FIWE 2.84 & 510 & FIWE 2.578 \\
\hline 467 & JIWE 2.160 & 511 & JIWE 2.554 \\
\hline 468 & JIWE 2.140 & 512 & fIWE 2.574 \\
\hline 469 & JIWE 2.48 & 513 & JIWE 2.545 \\
\hline 470 & JIWE 2.618 & 514 & FIWE 2.613 \\
\hline 471 & JIWE 2.151 & 515 & JIWE 2.588 \\
\hline 472 & JIWE 2.197 & 516 & FIWE 2.589 \\
\hline 473 & JIWE 2.155 & 517 & FIWE 2.590 \\
\hline 474 & fIWE 2.74 & 518 & FIWE 2.591 \\
\hline 475 & JIWE 2.5 & 519 & JIWE 2.593 \\
\hline 476 & JIWE 2.103 & 520 & FIWE 2.597 \\
\hline 477 & fIWE 2.7 & 521 & FIWE 2.594 \\
\hline 478 & JIWE 2.87 & 522 & FIWE 2.592 \\
\hline 479 & JIWE 2.565 & 523 & fIWE 2.577 \\
\hline 480 & JIWE 2.198 & 524 & FIWE 2.626.iii \\
\hline 481 & JIWE 2.199 & 525 & FIWE 2.599 \\
\hline 482 & JIWE 2.564 & 526 & JIWE 1.174 \\
\hline 483 & JIWE 2.180 & 527 & JIWE 2.619 \\
\hline 484 & JIWE 2.67 & 528 & fIWE 2.617 \\
\hline 485 & JIWE 2.61 & 529 & JIWE 2.626.iv \\
\hline 486 & JIWE 2.182 & 530 & fIWE 2.601 \\
\hline 487 & JIWE 2.141 & 531 & JIWE 2.602 \\
\hline 488 & JIWE 2.142 & 532 & JIWE 2.603 \\
\hline 489 & JIWE 2.143 & 533 & FIWE 1.18 \\
\hline 490 & JIWE 2.156 & 534 & JIWE 1.205 \\
\hline 491 & FIWE 2.144 & $534 \mathrm{a}$ & fIWE 1.15 \\
\hline 492 & FIWE 2.203.xix & 535 & JIWE 2.579 \\
\hline 493 & FIWE 2.203.ii & 536 & FIWE 2.581 \\
\hline
\end{tabular}




\begin{tabular}{|c|c|c|c|}
\hline 537 & JIWE 2.584 & 578 & JIWE 1.77 \\
\hline 538 & JIWE 2.585 & 579 & JIWE 1.47 \\
\hline 539 & FIWE 1.206 & 580 & fIWE 1.42 \\
\hline 540 & JIWE 2.582 & 581 & FIWE 1.59 \\
\hline 541 & FIWE 1.207 & 582 & FIWE 1.101 \\
\hline 542 & FIWE 2.614 & 583 & JIWE 1.69 \\
\hline 543 & FIWE 2.579 & 584 & JIWE 1.70 \\
\hline 544 & JIWE 2.586 & 585 & fIWE 1.94 \\
\hline 545 & FIWE 2.580 & 586 & JIWE 1.79 \\
\hline 546 & FIWE 1.208 & 587 & FIWE 1.53 \\
\hline 547 & FIWE 2.583 & 588 & JIWE 1.46 \\
\hline 548 & JIWE 2.587 & 589 & JIWE 1.44 \\
\hline 549 & FIWE 1.209 & 590 & FIWE 1.62 \\
\hline 550 & JIWE 1.210 & 591 & FIWE 1.66 \\
\hline $551 \mathrm{a}$ & FIWE 1.211 & 592 & FIWE 1.97 \\
\hline $551 \mathrm{~b}$ & JIWE 2.615 & 593 & FIWE 1.111 \\
\hline $551 \mathrm{c}$ & FIWE 1.212 & 594 & FIWE 1.48 \\
\hline $551 d$ & FIWE 1.213 & 595 & FIWE 1.75 \\
\hline $551 \mathrm{e}$ & JIWE 1.214 & 596 & FIWE 1.64 \\
\hline 552 & FIWE 1.19 & 597 & FIWE 1.71 \\
\hline 553 & FIWE 1.20 & 598 & FIWE 1.65 \\
\hline 554 & FIWE 1.21 & 599 & fIWE 1.61 \\
\hline 555 & JIWE 1.25 & 600 & fIWE 1.76 \\
\hline 556 & JIWE 1.26 & 601 & FIWE 1.78 \\
\hline 557 & JIWE 1.24 & 602 & FIWE 1.112 \\
\hline 558 & fIWE 1.27 & 603 & FIWE 1.102 \\
\hline 559 & FIWE 1.28 & 604 & FIWE 1.54 \\
\hline 560 & FIWE 1.29 & 605 & fIWE 1.83 \\
\hline 561 & FIWE 1.23 & 606 & JIWE 1.63 \\
\hline 562 & FIWE 1.215 & 607 & FIWE 1.85 \\
\hline 563 & FIWE 1.40 & 608 & JIWE 1.89 \\
\hline 564 & FIWE 1.216 & 609 & FIWE 1.80 \\
\hline 565 & JIWE 1.217 & 610 & fIWE 1.68 \\
\hline 566 & JIWE 1.218 & 611 & JIWE 1.86 \\
\hline 567 & FIWE 1.38 & 612 & fIWE 1.56 \\
\hline 568 & JIWE 1.36 & 613 & fIWE 1.87 \\
\hline 569 & fIWE 1.82 & 614 & JIWE 1.90 \\
\hline 570 & JIWE 1.84 & 615 & FIWE 1.67 \\
\hline 571 & JIWE 1.81 & 616 & FIWE 1.88 \\
\hline 572 & fIWE 1.57 & 617 & FIWE 1.100 \\
\hline 573 & JIWE 1.58 & 618 & FIWE 1.51 \\
\hline 574 & JIWE 1.49 & 619 & FIWE 1.73 \\
\hline 575 & fIWE 1.72 & $619 a$ & FIWE 1.113 \\
\hline 576 & FIWE 1.52 & $619 b$ & FIWE 1.114 \\
\hline 577 & JIWE 1.50 & $619 c$ & JIWE 1.115 \\
\hline
\end{tabular}




\begin{tabular}{|c|c|c|c|}
\hline $619 d$ & JIWE 1.116 & 653a (Frey) & JIWE 1.221 \\
\hline $619 \mathrm{e}$ & JIWE 1.219 & 653a (Lif.) & JIWE 1.155 \\
\hline 620 & JIWE 1.193 & $653 \mathrm{~b}$ & JIWE 1.157 \\
\hline 621 & JIWE 1.125 & 654 & JIWE 1.150 \\
\hline 622 & JIWE 1.126 & $654 a$ & FIWE 1.160 \\
\hline 623 & JIWE 1.122 & 655 & FIWE 1.cf.163-8 \\
\hline 624 & JIWE 1.123 & 656 & FIWE 1.196 \\
\hline 625 & JIWE 1.131 & 657 & JIWE 1.169 \\
\hline 626 & JIWE 1.132 & 658 & JIWE 1.170 \\
\hline 627 & JIWE 1.118 & 659 & JIWE 1.171 \\
\hline 628 & JIWE 1.119 & 660 & JIWE 1.172 \\
\hline 629 & JIWE 1.120 & $660 \mathrm{a}$ & JIWE 1.175 \\
\hline 630 & JIWE 1.121 & $660 \mathrm{~b}$ & JIWE 1.176 \\
\hline 631 & JIWE 1.124 & $660 \mathrm{c}$ & JIWE 1.185 \\
\hline 632 & JIWE 1.134 & $660 d$ & JIWE 1.187 \\
\hline 633 & JIWE 1.194 & 661 & JIWE 1.183 \\
\hline 634 & JIWE 1.195 & 662 & JIWE 1.180 \\
\hline 635 & JIWE 1.137 & 663 & JIWE 1.181 \\
\hline $635 a$ & JIWE 1.138 & 664 & JIWE 1.182 \\
\hline $635 b$ & JIWE 1.139 & 665 & JIWE 1.179 \\
\hline 636 & JIWE 1.11 & $665 a$ & JIWE 1.197 \\
\hline 637 & JIWE 1.203 & 666 & JIWE 1.200 \\
\hline 638 & JIWE 1.4 & 667 & JIWE 1.190 \\
\hline 639 & FIWE 1.5 & 668 & JIWE 1.198 \\
\hline 640 & FIWE 1.6; IfudO 3.Syr43 & 669 & JIWE 1.199 \\
\hline 641 & FIWE 1.202 & 670 & JIWE 1.189 \\
\hline 642 & JIWE 1.9 & 671 & JIWE 1.191 \\
\hline 643 & JIWE 1.7 & 672 & JIWE 1.192 \\
\hline $643 a$ & JIWE 1.8 & $672 \mathrm{a}$ & JIWE 1.226 \\
\hline 644 & JIWE 1.2 & 673 & fIWE 1.227 \\
\hline 645 & JIWE 1.201 & 674 & fIWE 1.228 \\
\hline 646 & JIWE 1.1 & 675 & IfudO 1.Pan2 \\
\hline 647 & JIWE 1.142 & 676 & IfudO 1.Pan 1 \\
\hline 648 & fIWE 1.141 & 677 & IfudO 1.Pan3 \\
\hline 649 & JIWE 1.3 & 678 & IfudO 1.Pan4 \\
\hline $649 \mathrm{a}$ & JIWE 1.143 & $678 \mathrm{a}$ & IfudO 1.Pan5 \\
\hline 650 & JIWE 1.145 & 679 & IfudO 1.p.1 \\
\hline $650 \mathrm{a}$ & fIWE 1.146 & 680 & IfudO 1.Dal2 \\
\hline $650 \mathrm{~b}$ & JIWE 1.147 & $680 \mathrm{a}$ & IfudO 1.Dal3 \\
\hline $650 \mathrm{c}$ & JIWE 1.148 & 681 & IfudO 1.Moes1 \\
\hline $650 d$ & JIWE 1.149 & $681 \mathrm{a}$ & IfudO 1.Thr5 \\
\hline $650 \mathrm{e}$ & JIWE 1.144 & $681 \mathrm{~b}$ & IfudO 1.App7 \\
\hline 651 & fIWE 1.151 & 682 & IfudO 1.BS1 \\
\hline 652 & JIWE 1.152 & 683 & IfudO 1.BS5 \\
\hline 653 & JIWE 1.220 & $683 a$ & IfudO 1.BS7 \\
\hline
\end{tabular}




\begin{tabular}{|c|c|c|c|}
\hline $683 b$ & IfudO 1.BS9 & 709 & IfudO 1.Ach 43 \\
\hline 684 & IfudO 1.BS4 & 710 & IfudO 1.Ach 42 \\
\hline 685 & IfudO 1.BS10 & 711 & IfudO 1.Ach44 \\
\hline 686 & IfudO 1.BS12 & $711 \mathrm{a}$ & IfudO 1.Ach46 \\
\hline 687 & IfudO 1.BS11 & $711 b$ & IfudO 1.Ach 45 \\
\hline 688 & IfudO 1.BS13 & 712 & IfudO 1.Ach28 \\
\hline 689 & $I f u d O$ 1.BS 15 & 713 & IfudO 1.Ach30 \\
\hline $689 a$ & IfudO 1.BS14 & 714 & IfudO 1.App10 \\
\hline 690 & IfudO 1.BS20 & 715 & IfudO 1.Ach34 \\
\hline $690 \mathrm{a}$ & IfudO 1.BS22 & $715 \mathrm{a}$ & IfudO 1.Ach26 \\
\hline 690b & IfudO 1.BS23 & $715 b$ & IfudO 1.Ach27 \\
\hline 691 & IfudO 1.BS17 & $715 c$ & IfudO 1.Ach33 \\
\hline $691 \mathrm{a}$ & IfudO 1.BS16 & $715 d$ & IfudO 1.Ach31 \\
\hline $691 b$ & IfudO 1.BS19 & $715 \mathrm{e}$ & IfudO 1.App12 \\
\hline 692 & IfudO 1.Thr3; IfudO 2.12 & $715 f$ & IfudO 1.Ach32 \\
\hline $692 \mathrm{a}$ & IfudO 1.Thr4; IfudO 2.13 & $715 \mathrm{~g}$ & IfudO 1.App13 \\
\hline 693 & IfudO 1.Mac16 & $715 \mathrm{~h}$ & IfudO 1.Ach29 \\
\hline $693 \mathrm{a}$ & IfudO 1.Mac17 & $715 \mathrm{i}$ & IfudO 1.Ach 40 \\
\hline $693 b$ & IfudO 1.Mac13 & 716 & IfudO 1.App2 \\
\hline $693 c$ & IfudO 1.Mac14 & 717 & IfudO 1.App21 \\
\hline $693 d$ & IfudO 1.App9 & 718 & IfudO 1.Ach 47 \\
\hline 694 & IfudO 1.Macl & $718 \mathrm{a}$ & IfudO 1.Ach48 \\
\hline $694 a$ & IfudO 1.Mac6 & 719 & $I f u d O$ 1.Ach51 \\
\hline $694 b$ & IfudO 1.Mac7 & 720 & IfudO 1.Ach54 \\
\hline 695 & IfudO 1.Ach24 & 721 & IfudO 1.Ach52 \\
\hline 696 & IfudO 1.Ach 16 & $721 \mathrm{a}$ & IfudO 1.Ach55 \\
\hline $696 \mathrm{a}$ & IfudO 1.Ach17 & $721 b$ & IfudO 1.Ach56 \\
\hline 696b & IfudO 1.Ach 18 & $721 \mathrm{c}$ & IfudO 1.Ach53 \\
\hline $696 c$ & IfudO 1.Ach 19 & 722 & IfudO 1.Ach58 \\
\hline 697 & IfudO 1.Ach6 & 723 & IfudO 1.Ach59 \\
\hline 698 & IfudO 1.Ach7 & 724 & IfudO 1.Aegina \\
\hline 699 & IfudO 1.Ach1 & $725 \mathrm{a}$ & IfudO 1.Ach70 \\
\hline 700 & IfudO 1.Ach1 & $725 b$ & IfudO 1.Ach71 \\
\hline 701 & IfudO 1.Ach1 & 726 & IfudO 1.Ach65 \\
\hline 702 & IfudO 1.Ach8 & 727 & IfudO 1.Ach60 \\
\hline 703 & IfudO 1.Ach3 & 728 & IfudO 1.Ach62 \\
\hline 704 & IfudO 1.Ach3 & 729 & IfudO 1.Ach63 \\
\hline 705 & IfudO 1.Ach3 & 730 & IfudO 1.Ach61 \\
\hline 706 & IfudO 1.Ach 10 & 731 & IfudO 1.Ach64 \\
\hline 707 & IfudO 1.Ach 12 & $731 \mathrm{a}$ & IfudO 1.Delos \\
\hline 708 & IfudO 1.Ach13 & $731 b$ & IfudO 1.App20 \\
\hline $708 \mathrm{a}$ & IfudO 1.Ach9 & $731 \mathrm{c}$ & IfudO 1.Cre3 \\
\hline $708 b$ & IfudO 1.Ach2 & $731 d$ & IfudO 1.Crel \\
\hline $708 c$ & IfudO 1.Ach23 & $731 \mathrm{e}$ & - \\
\hline $708 d$ & IfudO 1.Ach25 & $731 \mathrm{f}$ & IfudO 2.5 \\
\hline
\end{tabular}




\begin{tabular}{|c|c|c|c|}
\hline $731 \mathrm{~g}$ & IfudO 2.2 & 770 & IfudO 2.172 \\
\hline 732 & fIWE 2.596 & 771 & IfudO 2.169 \\
\hline $732 \mathrm{a}$ & JIWE 2.595 & 772 & IfudO 2.180 \\
\hline $733 \mathrm{St}$ & JIWE 2.552 & 773 & - \\
\hline $733 \mathrm{a}$ & JIWE 2.570 & 774 & IfudO 2.179 \\
\hline $733 b$ & JIWE 2.555 & 775 & IfudO 2.205 \\
\hline $733 c$ & JIWE 2.619 & 776 & IfudO 2.206 \\
\hline $733 d$ & JIWE 2.600 & 777 & IfudO 2.196 \\
\hline $733 \mathrm{e}$ & fIWE 2.546 \& 562 & 778 & IfudO 2.208 \\
\hline $733 \mathrm{f}$ & fIWE 2.629.ii & $779-80$ & - \\
\hline $733 \mathrm{~g}$ & JIWE 2.620 & 781 & IfudO 2.219 \\
\hline 734 & fIWE 2.598 & 782 & - \\
\hline 735 & IfudO 3.Сyp3 & 783 & IfudO 2.245 \\
\hline 736 & IfudO 3.Cyp1 & 784 & IfudO 2.244 \\
\hline 737 & IfudO 3.App23 & 785 & IfudO 2.232 \\
\hline 738 & IfudO 2.36 & 786 & IfudO 2.233 \\
\hline 739 & IfudO 2.41 & 787 & IfudO 2.234 \\
\hline 740 & IfudO 2.42 & 788 & IfudO 2.236 \\
\hline 741 & IfudO 2.43 & 789 & IfudO 2.235 \\
\hline 742 & $I f u d O 2.40$ & 790 & IfudO 2.237 \\
\hline 743 & IfudO 2.M2 & 791 & IfudO 2.238 \\
\hline 744 & IfudO 2.46 & 792 & IfudO 2.239 \\
\hline 745 & IfudO 2.32 & 793 & IfudO 2.240 \\
\hline 746 & IfudO 2.33 & 794 & IfudO 2.241 \\
\hline 747 & IfudO 2.35 & 795 & IfudO 2.231 \\
\hline 748 & IfudO 2.37 & 796 & IfudO 2.162 \\
\hline 749 & IfudO 2.21 & 797 & 一 \\
\hline 750 & IfudO 2.54 & 798 & IfudO 2.156 \\
\hline 751 & IfudO 2.53 & 799 & IfudO 2.157 \\
\hline 752 & IfudO 2.146 & 800 & IfudO 2.151 \\
\hline 753 & IfudO 2.48 & 801 & IfudO 2.150 \\
\hline 754 & IfudO 2.49 & 802 & - \\
\hline 755 & IfudO 2.47 & 803 & IfudO 3.Syr53 \\
\hline 756 & IfudO 2.25 & 804 & IfudO 3.Syr54 \\
\hline 757 & IfudO 2.223 & 805 & IfudO 3.Syr58 \\
\hline 758 & IfudO 2.221 & 806 & IfudO 3.Syr61 \\
\hline 759 & IfudO 2.214 & 807 & IfudO 3.Syr62 \\
\hline 760 & IfudO 2.173 & 808 & IfudO 3.Syr63 \\
\hline $761-63$ & - & 809 & IfudO 3.Syr64 \\
\hline 764 & IfudO 2.182 & 810 & IfudO 3.Syr65 \\
\hline 765 & - & 811 & IfudO 3.Syr66 \\
\hline 766 & IfudO 2.168 & 812 & IfudO 3.Syr59 \\
\hline 767 & - & 813 & IfudO 3.Syr55 \\
\hline 768 & IfudO 2.175 & 814 & IfudO 3.Syr60 \\
\hline 769 & IfudO 2.176 & 815 & IfudO 3.Syr70 \\
\hline
\end{tabular}




\begin{tabular}{|c|c|c|c|}
\hline 816 & IfudO 3.Syr68 & 865 & IfudO 3.App12 \\
\hline 817 & IfudO 3.Syr56 & $866-68$ & - \\
\hline 818 & IfudO 3.Syr57 & 869 & $I f u d O$ 3.Syr30 \\
\hline 819 & IfudO 3.Syr76 & 870 & $I f u d O$ 3.Syr28 \\
\hline 820 & IfudO 3.Syr49 & 871 & IfudO 3.Syr29 \\
\hline 821 & IfudO 3.Syr44 & 872 & IfudO 3.Syr31 \\
\hline 822 & IfudO 3.Syr46 & 873 & $I f u d O$ 3.Syr24 \\
\hline $822 n$ & IfudO 3.Syr47 & 874 & IfudO 3.Syr27 \\
\hline 823 & IfudO 3.Syr45 & 875 & IfudO 3.Syr20 \\
\hline 824 & IfudO 3.Syr48 & 876 & IfudO 3.p.24 \\
\hline 825 & IfudO 3.App22 & 877 & IfudO 3.p.24 \\
\hline 826 & IfudO 3.Syr83 & 878 & IfudO 3.Syr 12 \\
\hline 827 & IfudO 3.Syr82 & 879 & IfudO 3.Syr 1 \\
\hline $828 \mathrm{a}$ & IfudO 3.Syr85 & 880 & IfudO 3.Syr2 \\
\hline $828 b$ & IfudO 3.Syr84 & 881 & IfudO 3.p.1 \\
\hline 829 & IfudO 3.Syr86 & 882 & IfudO 2.246 \\
\hline 830 & IfudO 3.Syr88 & 883-909 & - \\
\hline 831 & IfudO 3.Syr87 & 910 & IfudO 2.251 \\
\hline 832 & IfudO 3.Syr 127 & $911-24$ & - \\
\hline 833 & IfudO 3.Syr 128 & 925 & IfudO 2.250 \\
\hline 834 & IfudO 3.Syr96 & $926-30$ & - \\
\hline 835 & IfudO 3.Syr98 & 931 & IfudO 2.249 \\
\hline 836 & IfudO 3.Syr97 & $932-53$ & - \\
\hline 837 & IfudO 3.Syr 100 & 954 & IfudO 2.4 \\
\hline 838 & IfudO 3.Syr 100 & $955-90$ & - \\
\hline 839 & IfudO 3.Syr 102 & 991 & IfudO 3.Syr5 \\
\hline 840 & IfudO 3.Syr 105 & 992-1009 & - \\
\hline 841 & IfudO 3.Syr 103 & 1010 & IfudO 3.Syr51 \\
\hline 842 & IfudO 3.Syr 104 & 1011 & IfudO 3.Syr52 \\
\hline 843 & IfudO 3.Syr94 & $1012-23$ & - \\
\hline 844 & IfudO 3.Syr 130 & 1024 & IfudO 3.App 1 \\
\hline 845 & IfudO 3.Syr91 & $1025-1232$ & - \\
\hline 846 & IfudO 3.App 13 & 1233 & IfudO 3.Syr22 \\
\hline 847 & IfudO 3.App 14 & $1234-1413$ & - \\
\hline 848 & IfudO 3.Syr41 & 1414 & IfudO 2.184 \\
\hline 849 & IfudO 3.p.66 & 1415 & IfudO 3.Syr78 \\
\hline 850 & IfudO 3.p.66 & 1416 & IfudO 3.Syr79 \\
\hline 851 & IfudO 3.p.66 & 1417 & IfudO 3.Syr80 \\
\hline 852 & IfudO 3.App 15 & 1418 & IfudO 3.Syr80 \\
\hline 853 & IfudO 3.Syr35 & 1419 & IfudO 3.App21 \\
\hline $854-60$ & - & $1420-23$ & - \\
\hline 861 & IfudO 3.Syr34 & 1424 & fIGRE 3 \\
\hline 862 & IfudO 3.p.231 & 1425 & fIGRE 4 \\
\hline 863 & IfudO 3.Syr37 & 1426 & JIGRE 5 \\
\hline 864 & IfudO 3.Syr38 & 1427 & fIGRE 1 \\
\hline
\end{tabular}




\begin{tabular}{|c|c|c|c|}
\hline 1428 & JIGRE 2 & 1473 & FIGRE 62 \\
\hline 1429 & JIGRE 6 & 1474 & FIGRE 63 \\
\hline 1430 & JIGRE 7 & 1475 & JIGRE 64 \\
\hline 1431 & fIGRE 8 & 1476 & JIGRE 65 \\
\hline 1432 & JIGRE 13 & 1477 & FIGRE 130 \\
\hline 1433 & fIGRE 9 & 1478 & FIGRE 137 \\
\hline 1434 & JIGRE 14 & 1479 & JIGRE 138 \\
\hline 1435 & JIGRE 127-128 & 1480 & FIGRE 66 \\
\hline 1436 & fIGRE 16 & 1481 & fIGRE 106 \\
\hline 1437 & JIGRE 17 & 1482 & FIGRE 107 \\
\hline 1438 & JIGRE 15 & 1483 & fIGRE 108 \\
\hline 1439 & fIGRE 21 & 1484 & FIGRE 109 \\
\hline 1440 & JIGRE 22 & 1485 & $\mathscr{J I G R E} 110$ \\
\hline 1441 & JIGRE 24 & 1486 & FIGRE 111 \\
\hline 1442 & JIGRE 25 & 1487 & FIGRE 112 \\
\hline 1443 & JIGRE 27 & 1488 & FIGRE 113 \\
\hline 1444 & JIGRE 28 & 1489 & JIGRE 114 \\
\hline 1445 & JIGRE 135 & 1490 & FIGRE 30 \\
\hline 1446 & JIGRE 19 & 1491 & FIGRE 67 \\
\hline 1447 & fIGRE 20 & 1492 & JIGRE 68 \\
\hline 1448 & JIGRE 136; IfudO 2.M5 & 1493 & FIGRE 69 \\
\hline 1449 & JIGRE 125 & 1494 & FIGRE 70 \\
\hline 1450 & FIGRE 129 & 1495 & FIGRE 71 \\
\hline 1451 & JIGRE 29 & 1496 & JIGRE 72 \\
\hline 1452 & FIGRE 41 & 1497 & FIGRE 73 \\
\hline 1453 & fIGRE 42 & 1498 & JIGRE 74 \\
\hline 1454 & JIGRE 43 & 1499 & fIGRE 75 \\
\hline 1455 & JIGRE 44 & 1500 & JIGRE 76 \\
\hline 1456 & JIGRE 45 & 1501 & FIGRE 77 \\
\hline 1457 & JIGRE 46 & 1502 & FIGRE 78 \\
\hline 1458 & fIGRE 47 & 1503 & JIGRE 79 \\
\hline 1459 & $\mathscr{J I G R E} 48$ & 1504 & JIGRE 80 \\
\hline 1460 & JIGRE 49 & 1505 & fIGRE 81 \\
\hline 1461 & fIGRE 50 & 1506 & FIGRE 82 \\
\hline 1462 & JIGRE 51 & 1507 & FIGRE 83 \\
\hline 1463 & JIGRE 52 & 1508 & fIGRE 31 \\
\hline 1464 & fIGRE 53 & 1509 & FIGRE 32 \\
\hline 1465 & JIGRE 54 & 1510 & fIGRE 33 \\
\hline 1466 & JIGRE 55 & 1511 & FIGRE 34 \\
\hline 1467 & JIGRE 56 & 1512 & FIGRE 35 \\
\hline 1468 & JIGRE 57 & 1513 & fIGRE 36 \\
\hline 1469 & JIGRE 58 & 1514 & fIGRE 84 \\
\hline 1470 & JIGRE 59 & 1515 & JIGRE 85 \\
\hline 1471 & FIGRE 60 & 1516 & FIGRE 86 \\
\hline 1472 & fIGRE 61 & 1517 & FIGRE 87 \\
\hline
\end{tabular}




\begin{tabular}{|c|c|c|c|}
\hline 1518 & JIGRE 88 & $10 *$ & JIWE 2.610 \\
\hline 1519 & JIGRE 89 & $11^{*}$ & fIWE 2.372 \\
\hline 1520 & fIGRE 90 & $12^{*}-14 *$ & - \\
\hline 1521 & fIGRE 91 & $15^{*}$ & JIWE 2.252 \\
\hline 1522 & fIGRE 37 & $16^{*}-23^{*}$ & - \\
\hline 1523 & fIGRE 92 & $24 *$ & FIWE 2.40 \\
\hline 1524 & JIGRE 93 & $25^{*}$ & JIWE 2.148 \\
\hline 1525 & JIGRE 94 & $26 *-27 *$ & - \\
\hline 1526 & fIGRE 95 & $28 *$ & JIWE 2.606 \\
\hline 1527 & fIGRE 96 & $29 *$ & - \\
\hline 1528 & JIGRE 97 & $30 *$ & fIWE 2.122 \\
\hline 1529 & fIGRE 98 & $31 *$ & JIWE 2.145 \\
\hline 1530 & FIGRE 38 & $32 *$ & fIWE 2.104 \\
\hline 1531 & FIGRE 115 & $33^{*}$ & JIWE 2.78 \\
\hline 1532 & FIGRE 116 & $34^{*}$ & - \\
\hline 1533 & FIGRE 118 & $35^{*}$ & fIWE 2.105 \\
\hline 1534 & FIGRE 119 & $36^{*}$ & JIWE 2.605 \\
\hline 1535 & FIGRE 120 & $37 *$ & fIWE 2.604 \\
\hline 1536 & FIGRE 133 & $38^{*}$ & JIWE 2.607 \\
\hline 1537 & FIGRE 121 & $39 *$ & JIWE 2.149 \\
\hline 1538 & FIGRE 122 & $40 *$ & JIWE 2.150 \\
\hline 1539 & FIGRE 40 & $41 *$ & JIWE 2.203.ix \\
\hline $1 *-4 *$ & - & $42 *-77 *$ & - \\
\hline $5^{*}$ & FIWE 2.341 & $78^{*}$ & IfudO 1.BS21 \\
\hline $6 *-7 *$ & 一 & $79 *-80 *$ & - \\
\hline $8^{*}$ & FIWE 2.400 & $81 *$ & JIWE 2.401 \\
\hline $9 *$ & JIWE 2.609 & $82 *-103 *$ & - \\
\hline
\end{tabular}

